\title{
Antepartum Obstetric Air Embolism
}

National Cancer Institute

\section{Source}

National Cancer Institute. Antepartum Obstetric Air Embolism. NCI Thesaurus. Code C111780.

Presence of a large air bubble in the maternal vascular system which orig inated from a distant site during the antepartum period. 